\title{
The Sodium Pump: Novel Functions in the Brain
}

\section{Heng-Ye Man*}

Department of Biology, Boston University, 5 Cummington Mall, Boston, MA 02215, USA

The $\mathrm{Na}^{+}, \mathrm{K}^{+}$-ATPase (NKA), i.e. sodium pump, is a plasma membrane-embedded protein that maintains the asymmetric distribution of sodium and potassium. In neurons, because of the frequent perturbation of ion homeostasis due to constant synaptic and neuronal activity, the workload of NKA is so high that it consumes half of the ATP produced in the brain [1]. For every ATP hydrolyzed, Three $\mathrm{Na}^{+}$from the cytosolic side and two $\mathrm{K}^{+}$from the extracellular medium are exchanged, making the NKA electrogenic, and helping the formation of negative resting potential [2]. The sodium gradient, not only forms the basis of excitation, but is also harnessed by many secondary transport systems, including glutamate transporters and the $\mathrm{Na}^{+}-\mathrm{Ca}^{+}$exchanger. NKA is a heterodimer composed of two subunits, $\alpha$ and $\beta$, in which $\alpha$ is the catalytic subunit, containing ATPase activity and $\beta$ is a regulatory subunit, required for the enzymatic activity of NKA $[3,4]$. The recently discovered $\gamma$ subunit, associates with the NKA complex but its function remain elusive.

So far, $4 \alpha$ and $3 \beta$ subunits have been cloned in mammals. The different subunit composition and cellular/subcellular distribution of NKA, may confer cell type specific properties. $\alpha 1$ is ubiquitously distributed and $\alpha 4$ seems to be only expressed in testis. $\alpha 1-3$ are all abundantly expressed in the brain [2]. At the single neuron level, immunostainings have shown widespread localization of NKA in the soma as well the dendrites, but its subcellular distribution has not been carefully examined $[5,6]$. By double staining with the synaptic marker PSD-95, we find an enrichment of the NKA $\alpha 1$ subunit, at synaptic sites in cultured cortical neurons [7]. In addition, consistent with other reports [5], our biochemical analysis using membrane fractionation, also confirmed the existence of NKA in purified synaptosomes from brain tissue, indicating the synaptic localization of NKA, in both cultured neurons and native brain. Although NKA biology has been extensively studied in peripheral tissues such as cardiac and renal epithelial cells, few studies have been done on its role in neurons.

In the brain, neuronal communication is achieved mainly via glutamate-mediated synaptic transmission. There are two major types of ionotropic glutamate receptors - AMPA receptors (AMPARs) and NMDA receptors (NMDARs). AMPARs mediate the vast majority of fast excitatory synaptic transmission, whereas NMDARs play vital role in the modulation of synaptic efficacy, generating synaptic plasticity. AMPARs are heterotetromers, assembled from different combinations of four subunits GluA1-4, the most common of which are receptors containing GluA1/GluA2 or GluAR2/GluA3. NMDARs are composed of GluN1 subunits, and at least one GluN2 subunit, out of four GluN2 subtypes GluN2A-2D. AMPAR and NMDAR colocalize at the postsynaptic domain at a high density, an accumulation believed to be stabilized and regulated, by interaction with cytosolic scaffolding proteins. These AMPAR-associated proteins usually contain one or more PSD domains, through which they interact with the intracellular C-terminus of GluAs, to regulate AMPAR synaptic targetting, intracellular trafficking as well as channel function $[8,9]$. AMPARs are primarily sodium channels. In comparison, NMDARs permit both sodium and calcium, with the latter playing an important role in synaptic plasticity, as calcium triggers a variety of downstream signaling events. However, at resting conditions, NMDARs are blocked by extracellular magnesium trapped in the channel pore, which can only be relieved by membrane depolarization. During action potentialtriggered glutamate release, sodium influx via AMPARs depolarizes the plasma membrane, leading to the release of $\mathrm{Mg}^{2+}$ from the channel pore and thus, activation of the NMDARs.

Given the dense synaptic expression of sodium-permeant glutamate receptors, whose persistent activity is directly coupled to the function of NKA; it is reasonable to assume the existence of crosstalk between the sodium-permeating channels and NKA for functional coordination. Indeed, we find that NKA is physically associated with AMPARs. Inhibition of NKA by Ouabain, induces a rapid translocation of AMPARs from the plasma membrane to the cytosolic domain, where they are digested via proteasome-mediated degradation, presumably, as a result of receptor ubiquitination [10,11], causing a lasting suppression of synaptic transmission [7]. This suggests a homeostatic regulation of AMPARs by NKA, i.e. the intracellular sodium accumulation caused by NKA inactivity, leads to a removal of sodium-permeating channels at the cell-surface. It may explain the observation that in contrast to its toxicity, low doses of ouabain actually offer protection against neuronal death [12]. Because NKA starts losing function, at the early phase of stroke/ischemia due to the lack of ATP supply, the resulting rapid removal of surface AMPARs may help to alleviate the excitotoxicity. In support of this notion, ouabain has recently been suggested in the treatment of stroke-induced neuronal death [13].

Although NKA has been traditionally considered a pure energy transducing ion pump, evidence from recent years reveals that it is also involved in the regulation of many cellular functions including gene expression, cell growth, and cell motility. Protein-protein interactions are a major means of bridging NKA activity to signaling molecules or organelles [14-17]. For instance, the tyrosine kinase Src, which regulates a large number of cellular functions, including glutamatergic NMDA receptor channel property and synaptic plasticity $[18,19]$, associates with NKA via direct protein interaction. Interestingly, ouabain increases Src binding to NKA and elevates Src tyrosine kinase activity $[20,21]$. In a similar manner, the p85 subunit of phosphoinositide-3 kinase (PI3K) binds to the catalytic subunit of NKA through its SH3 domain, leading to the activation of PI3K and endocytosis of NKA in renal epithelial cells [22]. NKAs are functionally linked to intracellular calcium stores, through interaction with the inositol 1,4,5-triphosphate receptor (IP3R). This association activates IP3R, causing slow calcium oscillations and the activation of the transcription factor NF- $\mathrm{kB}$ [23]. In addition, NKA is also involved in the cell adhesion process by two means: the $\beta 2$ subunit itself functions as an adhesion molecule in the

*Corresponding author: Heng-Ye Man, Department of Biology, Boston University, 5 Cummington Mall, Boston, MA 02215, USA, E-mail: hman@bu.edu

Received September 06, 2012; Accepted September 06, 2012; Published September 11, 2012

Citation: Man HY (2012) The Sodium Pump: Novel Functions in the Brain Biochem Anal Biochem 1:e116. doi:10.4172/2161-1009.1000e116

Copyright: (c) 2012 Man HY. This is an open-access article distributed under the terms of the Creative Commons Attribution License, which permits unrestricted use, distribution, and reproduction in any medium, provided the original author and source are credited. 
brain, capable of promoting neurite growth in vitro [24]. Also, when bound with ouabain, NKA increases the expression of the cell adhesion molecule Connexin 32 and facilitates its cell surface expression $[25,26]$. All these indicate that, in addition to being an active ion transporter, NKA also functions as a transducer of signals from the extracellular milieu to the interior of the cell.

Ouabain as a specific, high affinity inhibitor of NKA has been used for the treatment of heart problems and in research for many years. The long-standing question is, whether there exists endogenous ouabain or other types of ligand molecules. Investigations have confirmed that, the same ouabain can be synthesized and released by the peripheral organ adrenal cortex and in the brain, mainly by the hypothalamus. Ouabain is therefore, now regarded as a bonafide physiological steroid hormone circulating the body and the nervous system [27,28]. Peripheral release of endogenous ouabain can be regulated by physical exercise, causing a rapid rise in concentration that declines rapidly upon rest [29]. Furthermore, half of the population with hypertension show augmented concentration of endogenous ouabain, suggesting a role in cardiovascular regulation [30-33]. The finding that Agrin specifically binds to NKA in neurons, provided another type of endogenous NKA ligand [34]. Agrin, originally found in the neuromuscular junction (NMJ), is released at the nerve terminals of motor neurons, and acts to recruit and enrich acetylcholine receptors on the postsynaptic end $[34,35]$. Since, agrin is also synthesized and released by neurons in the brain [36-38], a large amount of effort has been made to confirm a similar role for brain agrin, in recruiting glutamate receptors in central synapses. Findings, however, have turned out to be negative. No gross defects were observed in neural agrin mutant mice, suggesting a different function for brain agrin $[39,40]$. Interestingly, a study demonstrated that agrin binds specifically to a 3 subunits of NKA and inhibits neuronal NKA activity. A competition experiment confirms that, endogenous neuron-released agrin indeed regulates NKA function [36]. The physiological roles for endogenous NKA inhibitors in brain function remain to be investigated.

\section{References}

1. Foo K, Blumenthal L, Man HY (2012) Regulation of neuronal bioenergy homeostasis by glutamate. Neurochem Int 61: 389-396

2. Mobasheri A, Avila J, Cózar-Castellano I, Brownleader MD, Trevan M, et al. (2000) $\mathrm{Na}^{+}, \mathrm{K}^{+}-\mathrm{ATPase}$ isozyme diversity; comparative biochemistry and physiological implications of novel functional interactions. Biosci Rep 51-91.

3. Jaitovich AA, Bertorello AM (2006) $\mathrm{Na}^{+}, \mathrm{K}^{+}$-ATPase: an indispensable ion pumping-signaling mechanism across mammalian cell membranes. Semin Nephrol 26: 386-392.

4. Pierre SV, Xie Z (2006) The Na,K-ATPase receptor complex: its organization and membership. Cell Biochem Biophys 46: 303-316.

5. Anupama Adya HV, Mallick BN (1998) Comparison of Na-K ATPase activity in rat brain synaptosome under various conditions. Neurochem Int 33: 283-286.

6. Brines ML, Robbins RJ (1993) Cell-type specific expression of $\mathrm{Na}^{+}, \mathrm{K}\left({ }^{+}\right)$ATPase catalytic subunits in cultured neurons and glia: evidence for polarized distribution in neurons. Brain Res 631: 1-11.

7. Zhang D, Hou Q, Wang M, Lin A, Jarzylo L, et al. (2009) Na, K-ATPase activity regulates AMPA receptor turnover through proteasome-mediated proteolysis. J Neurosci 29: 4498-4511.

8. Man HY (2011) GluA2-lacking, calcium-permeable AMPA receptors - inducers of plasticity? Curr Opin Neurobiol 21: 291-298.

9. Man HY, Ju W, Ahmadian G, Wang YT (2000) Intracellular trafficking of AMPA receptors in synaptic plasticity. Cell Mol Life Sci 57: 1526-1534.

10. Hou Q, Gilbert J, Man HY (2011) Homeostatic regulation of AMPA receptor trafficking and degradation by light-controlled single-synaptic activation. Neuron 72: 806-818.
11. Lin A, Hou Q, Jarzylo L, Amato S, Gilbert J, et al. (2011) Nedd4-mediated AMPA receptor ubiquitination regulates receptor turnover and trafficking. $J$ Neurochem 27-39.

12. Golden WC, Martin LJ (2006) Low-dose ouabain protects against excitotoxic apoptosis and up-regulates nuclear Bcl-2 in vivo. Neuroscience 137: 133-144.

13. Wang JK, Portbury S, Thomas MB, Barney S, Ricca DJ, et al. (2006) Cardiac glycosides provide neuroprotection against ischemic stroke: discovery by a brain slice-based compound screening platform. Proc Natl Acad Sci U S A 103 10461-10466.

14. Aizman O, Aperia A (2003) Na,K-ATPase as a signal transducer. Ann N Y Acad Sci 986: 489-496.

15. Liu J (2005) Ouabain-induced endocytosis and signal transduction of the $\mathrm{Na} / \mathrm{K}$ ATPase. Front Biosci 10: 2056-2063.

16. Wang $\mathrm{H}$, Haas M, Liang M, Cai T, Tian J, et al. (2004) Ouabain assembles signaling cascades through the caveolar $\mathrm{Na}^{+} / \mathrm{K}^{+}$-ATPase. J Biol Chem 279 17250-17259.

17. Xie Z, Cai T (2003) $\mathrm{Na}^{+}-\mathrm{K}^{+}--$ATPase-mediated signal transduction: from protein interaction to cellular function. Mol Interv 3: 157-168.

18. Yu XM, Salter MW (1998) Gain control of NMDA-receptor currents by intracellular sodium. Nature 396: 469-474.

19. Yu XM, Salter MW (1999) Src, a molecular switch governing gain control of synaptic transmission mediated by $\mathrm{N}$-methyl-D-aspartate receptors. Proc Nat Acad Sci U S A 96: 7697-7704.

20. Haas M, Wang H, Tian J, Xie Z (2002) Src-mediated inter-receptor cross-talk between the $\mathrm{Na}+/ \mathrm{K}+-\mathrm{ATP}$ ase and the epidermal growth factor receptor relays the signal from ouabain to mitogen-activated protein kinases. J Biol Chem 277 : 18694-18702.

21. Wang XQ, Yu SP (2005) Novel regulation of Na, K-ATPase by Src tyrosine kinases in cortical neurons. J Neurochem 93: 1515-1523.

22. Yudowski GA, Efendiev R, Pedemonte CH, Katz Al, Berggren PO, et al. (2000) Phosphoinositide-3 kinase binds to a proline-rich motif in the $\mathrm{Na}+, \mathrm{K}+-\mathrm{ATPase}$ alpha subunit and regulates its trafficking. Proc Natl Acad Sci U S A 97: 65566561.

23. Zhang S, Malmersjö S, Li J, Ando H, Aizman O, et al. (2006) Distinct role of the $\mathrm{N}$-terminal tail of the Na,K-ATPase catalytic subunit as a signal transducer. Biol Chem 281: 21954-21962.

24. Müller-Husmann G, Gloor S, Schachner M (1993) Functional characterization of beta isoforms of murine Na,K-ATPase. The adhesion molecule on glia (AMOG/beta 2), but not beta 1, promotes neurite outgrowth. J Biol Chem 268 : 26260-26267.

25. Contreras RG, Flores-Maldonado C, Lázaro A, Shoshani L, Flores-Benitez D, et al. (2004) Ouabain binding to $\mathrm{Na}+, \mathrm{K}+-\mathrm{ATP}$ ase relaxes cell attachment and sends a specific signal (NACos) to the nucleus. J Membr Biol 198: 147-158.

26. Larre I, Ponce A, Fiorentino R, Shoshani L, Contreras RG,et al. (2006) Contacts and cooperation between cells depend on the hormone ouabain. Proc Natl Acad Sci U S A 103: 10911-10916.

27. Schoner W (2000) Ouabain, a new steroid hormone of adrenal gland and hypothalamus. Exp Clin Endocrinol Diabetes 108: 449-454

28. Schoner W (2002) Endogenous cardiac glycosides, a new class of steroid hormones. Eur J Biochem 269: 2440-2448.

29. Antolovic R, Bauer N, Mohadjerani M, Kost H, Neu H, et al. (2000) Endogenous ouabain and its binding globulin: effects of physical exercise and study on the globulin's tissue distribution. Hypertens Res 23: S93-S98.

30. Manunta P, Hamilton J, Rogowski AC, Hamilton BP, Hamlyn JM (2000) Chronic hypertension induced by ouabain but not digoxin in the rat: antihypertensive effect of digoxin and digitoxin. Hypertens Res 23: S77-S85.

31. Wang JG, Staessen JA, Messaggio E, Nawrot T, Fagard R (2003) Salt, endogenous ouabain and blood pressure interactions in the general population. J Hypertens 21: 1475-1481.

32. Yuan C, Manunta P, Chen S, Hamlyn JM, Haddy FJ, et al. (1993) Role of ouabain-like factors in hypertension: effects of ouabain and certain endogenous ouabain-like factors in hypertension. J Cardiovasc Pharmacol 22: S10-12.

33. Hilgenberg LG, Ho KD, Lee D, O'Dowd DK, Smith MA (2002) Agrin regulates 
neuronal responses to excitatory neurotransmitters in vitro and in vivo. Mol Cell Neurosci 19: 97-110.

34. Gillespie SK, Balasubramanian S, Fung ET, Huganir RL (1996) Rapsyn clusters and activates the synapse-specific receptor tyrosine kinase MuSK. Neuron 16: 953-962.

35. Wallace BG, Qu Z, Huganir RL (1991) Agrin induces phosphorylation of the nicotinic acetylcholine receptor. Neuron 6: 869-878.

36. Hilgenberg LG, Su H, Gu H, O'Dowd DK, Smith MA (2006) Alpha3Na ${ }^{+} / \mathrm{K}^{+}$ ATPase is a neuronal receptor for agrin. Cell 125: 359-369.

37. Mantych KB, Ferreira A (2001) Agrin differentially regulates the rates of axonal and dendritic elongation in cultured hippocampal neurons. J Neurosci 21: 6802 6809 .

38. Smith MA, Hilgenberg LG (2002) Agrin in the CNS: a protein in search of a function? Neuroreport 13: 1485-1495.

39. Li Z, Hilgenberg LG, O'Dowd DK, Smith MA (1999) Formation of functional synaptic connections between cultured cortical neurons from agrin-deficient mice. J Neurobiol 39: 547-557.

40. Serpinskaya AS, Feng G, Sanes JR, Craig AM (1999) Synapse formation by hippocampal neurons from agrin-deficient mice. Dev Biol 205: 65-78. 